\title{
Ground-State Properties of the Spin-1/2 Heisenberg-Ising Bond Alternating Chain with Dzyaloshinskii-Moriya Interaction
}

\author{
J. StrreČKA ${ }^{a}$, L. GÁlisová ${ }^{b}$ AND O. DerzhKO ${ }^{c}$ \\ ${ }^{a}$ Department of Theoretical Physics and Astrophysics, Faculty of Science, P.J. Šafárik University \\ Park Angelinum 9, 04001 Košice, Slovak Republic \\ ${ }^{b}$ Department of Applied Mathematics, Faculty of Mechanical Engineering, Technical University \\ Letná 9, 04200 Košice, Slovak Republic \\ ${ }^{c}$ Institute for Condensed Matter Physics, National Academy of Sciences of Ukraine \\ 1 Svientsitskii Str., L'viv-11, 79011, Ukraine
}

\begin{abstract}
Ground-state energy is exactly calculated for the spin-1/2 Heisenberg-Ising bond alternating chain with the Dzyaloshinskii-Moriya interaction. Under certain condition, which relates a strength of the Ising, Heisenberg and Dzyaloshinskii-Moriya interactions, the ground-state energy exhibits an interesting nonanalytic behavior accompanied with a gapless excitation spectrum.
\end{abstract}

PACS numbers: 05.50.+q, 05.70.Jk, 64.60.De, 75.10.Pq

\section{Introduction}

Quantum spin chains provide an excellent playground for theoretical studies of collective quantum phenomena as they may exhibit numerous exotic ground states and quantum critical points [1]. The spin-1/2 HeisenbergIsing bond alternating chain, which has been originally invented by Lieb et al. [2] and recently re-examined by Yao et al. [3], represents a valuable example of rigorously solved quantum spin chain. The present work aims to provide a generalization of this simple but nontrivial quantum spin model by taking into account the antisymmetric Dzyaloshinskii-Moriya interaction.

\section{Heisenberg-Ising chain}

Let us consider a bond alternating chain of $2 N$ spins $1 / 2$ with nearest-neighbor antiferromagnetic interactions, which are alternatively of the Heisenberg and Ising type, respectively. The total Hamiltonian of the model under consideration is given by

$$
\begin{aligned}
H & =\sum_{n=1}^{N}\left[J_{\mathrm{H}}\left(s_{2 n-1}^{x} s_{2 n}^{x}+s_{2 n-1}^{y} s_{2 n}^{y}+\Delta s_{2 n-1}^{z} s_{2 n}^{z}\right)\right. \\
& \left.+D\left(s_{2 n-1}^{x} s_{2 n}^{y}-s_{2 n-1}^{y} s_{2 n}^{x}\right)+2 J_{\mathrm{I}} s_{2 n}^{z} s_{2 n+1}^{z}\right],
\end{aligned}
$$

where the parameter $J_{\mathrm{H}}(\Delta)$ denotes the $X X Z$ Heisenberg interaction between $2 n-1$ and $2 n$ spins, $\Delta$ is an anisotropy in this interaction, and the parameter $D$ stands for the $z$ component of the antisymmetric Dzyaloshinskii-Moriya interaction present along the Heisenberg bonds. Furthermore, the term $2 J_{\text {I }}$ denotes the Ising interaction between $2 n$ and $2 n+1$ spins and the periodic boundary condition $s_{2 N+1}^{\alpha} \equiv s_{1}^{\alpha}(\alpha=x, y, z)$ is imposed for convenience.

First, let us eliminate from the Hamiltonian (1) the Dzyaloshinskii-Moriya term after performing a spin coordinate transformation. The spin rotation about the $z$-axis by the specific angle $\tan \varphi=D / J_{\mathrm{H}}$, which is performed at all even sites $2 n(n=1, \ldots, N)$,

$$
\begin{aligned}
& s_{2 n}^{x} \rightarrow s_{2 n}^{x} \cos \varphi+s_{2 n}^{y} \sin \varphi, \\
& s_{2 n}^{y} \rightarrow-s_{2 n}^{x} \sin \varphi+s_{2 n}^{y} \cos \varphi,
\end{aligned}
$$

ensures a precise mapping equivalence between the Hamiltonian (1) and the Hamiltonian

$$
\begin{aligned}
H & =\sum_{n=1}^{N}\left[\sqrt{J_{\mathrm{H}}^{2}+D^{2}}\left(s_{2 n-1}^{x} s_{2 n}^{x}+s_{2 n-1}^{y} s_{2 n}^{y}\right)\right. \\
& \left.+J_{\mathrm{H}} \Delta s_{2 n-1}^{z} s_{2 n}^{z}+2 J_{\mathrm{I}} s_{2 n}^{z} s_{2 n+1}^{z}\right] .
\end{aligned}
$$

From here onward, one may closely follow the rigorous procedure developed in Refs. [2,3]. According to this, the Hamiltonian (2) is rewritten in terms of raising and lowering operators in the subspace where the ground state is, and subsequently, the Jordan-Wigner transformation is applied to express the relevant spin Hamiltonian as a bilinear form of the Fermi operators. The Fourier and Bogolyubov transformations are finally employed to bring the Hamiltonian relevant for the ground-state properties into the diagonal form

$$
H=-\frac{N}{4} J_{\mathrm{H}} \Delta+\sum_{k} \Lambda_{k}\left(\beta_{k}^{\dagger} \beta_{k}-\frac{1}{2}\right),
$$

where 


$$
\Lambda_{k}=\sqrt{\left(\sqrt{J_{\mathrm{H}}^{2}+D^{2}}+J_{\mathrm{I}}\right)^{2}-4 \sqrt{J_{\mathrm{H}}^{2}+D^{2}} J_{\mathrm{I}} \cos ^{2} \frac{k}{2}} .
$$

From Eqs. (3) and (4) one easily finds the exact result for the ground-state energy of the antiferromagnetic spin- $1 / 2$ Heisenberg-Ising bond alternating chain (1) for $N \rightarrow \infty$ :

$$
\frac{E_{0}}{N}=-\frac{1}{4} J_{\mathrm{H}} \Delta-\frac{\sqrt{J_{\mathrm{H}}^{2}+D^{2}}+J_{\mathrm{I}}}{\pi} \mathrm{E}(a),
$$

where $\mathrm{E}(a)=\int_{0}^{\frac{\pi}{2}} \mathrm{~d} \theta \sqrt{1-a^{2} \sin ^{2} \theta}$ is the complete elliptic integral of the second kind with the modulus $a$,

$$
a^{2}=\frac{4 \sqrt{J_{\mathrm{H}}^{2}+D^{2}} J_{\mathrm{I}}}{\left(\sqrt{J_{\mathrm{H}}^{2}+D^{2}}+J_{\mathrm{I}}\right)^{2}} \geq 0 .
$$

Recall that the complete elliptic integral of the second kind is a nonanalytic function of its modulus for $a^{2}=1-\left(a^{\prime}\right)^{2} \approx 1$, i.e., $\mathrm{E}(a)-1 \propto \ln a^{\prime}\left(a^{\prime}\right)^{2}$. The condition $a^{2}=1$ holds just if $J_{\mathrm{I}}=\sqrt{J_{\mathrm{H}}^{2}+D^{2}}$ and hence, one may expect nonanalytic behavior of the ground-state energy (5) under this special constraint, which relates a strength of the Ising, Heisenberg and DzyaloshinskiiMoriya interactions.

Before proceeding to a more detailed discussion of the most interesting results, it is worthy to mention that our exact results correctly reproduce (in an absence of the Dzyaloshinskii-Moriya term) the results previously reported by Lieb et al. [2] for the isotropic version and by Yao et al. [3] for the anisotropic version of the antiferromagnetic spin-1/2 Heisenberg-Ising bond alternating chain. For simplicity, our subsequent analysis will be restricted just to a particular case of the model with the isotropic Heisenberg interaction $(\Delta=1)$, which exhibits all general features notwithstanding this limitation.

\section{Results and discussion}

In Fig. 1 we depict the elementary excitation energy spectrum $\Lambda_{k}$ calculated from Eq. (4) for two different values of the ratio $J_{\mathrm{I}} / J_{\mathrm{H}}$ and several values of the Dzyaloshinskii-Moriya anisotropy $D / J_{\mathrm{H}}$. Generally, the excitations are gapped with exception of the particular cases that satisfy the condition $J_{\mathrm{I}}=\sqrt{J_{\mathrm{H}}^{2}+D^{2}}$. The gapless excitation spectrum might be consequently found just if $J_{\mathrm{I}} / J_{\mathrm{H}} \geq 1$, which means that the Ising interaction must be at least twice as large as the Heisenberg one. If $D / J_{\mathrm{H}}=0$ is assumed, the system has gapless excitation spectrum for $J_{\mathrm{I}} / J_{\mathrm{H}}=1 \mathrm{in}$ accordance with the previously published results $[2,3]$. Interestingly, the gapless excitation spectrum emerges at higher values of the ratio $J_{\mathrm{I}} / J_{\mathrm{H}}$ regardless of the exchange anisotropy $\Delta$ whenever the Dzyaloshinskii-Moriya anisotropy is raised from zero.

The three-dimensional plot of the ground-state energy (5) is depicted in Fig. 2 as a function of the ratio $J_{\mathrm{I}} / J_{\mathrm{H}}$ between the Ising and Heisenberg interaction, as well as a relative strength of the Dzyaloshinskii-Moriya anisotropy $D / J_{\mathrm{H}}$. Referring to this plot, the ground-state energy monotonically decreases upon strengthening the ratio $J_{\mathrm{I}} / J_{\mathrm{H}}$ and/or the Dzyaloshinskii-Moriya

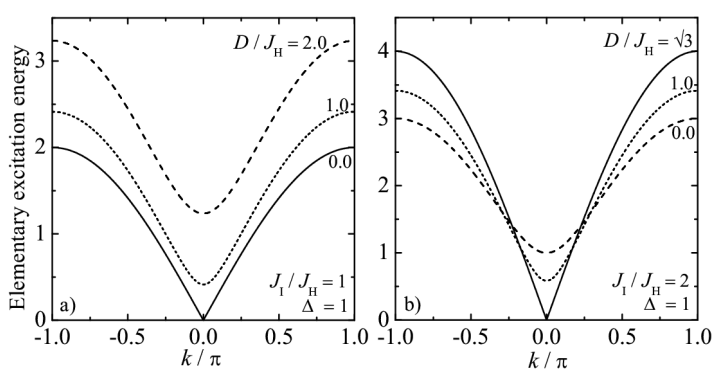

Fig. 1. Elementary excitation spectrum for several values of the Dzyaloshinskii-Moriya term $D / J_{\mathrm{H}}, \Delta=1$ and two different values of the ratio: (a) $J_{\mathrm{I}} / J_{\mathrm{H}}=1$, (b) $J_{\mathrm{I}} / J_{\mathrm{H}}=2$.

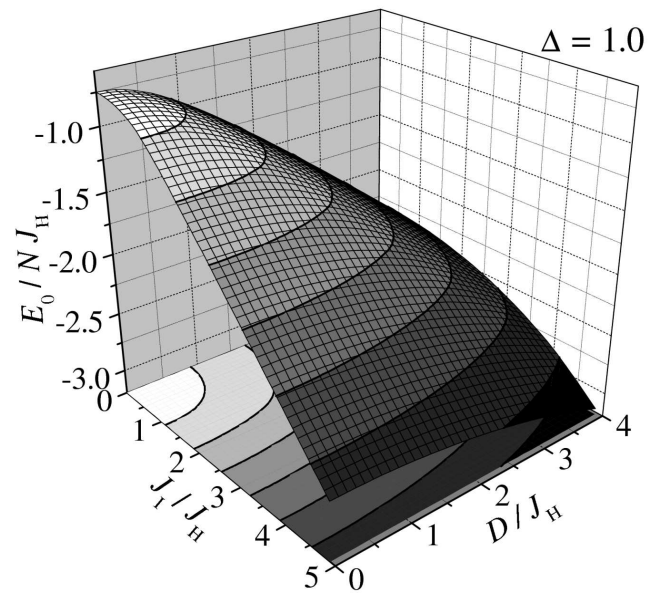

Fig. 2. Ground-state energy as a function of the Dzyaloshinskii-Moriya anisotropy $D / J_{\mathrm{H}}$ and the interaction ratio $J_{\mathrm{I}} / J_{\mathrm{H}}$ for the anisotropy parameter $\Delta=1$.

term $D / J_{\mathrm{H}}$. In accordance with this statement, the ground-state energy $E_{0} / N J_{\mathrm{H}}=-3 / 4$ of a system of the isolated Heisenberg dimers, which is achieved in the limit $J_{\mathrm{I}} / J_{\mathrm{H}} \rightarrow 0$ and $D / J_{\mathrm{H}} \rightarrow 0$, represents an upper bound for the ground-state energy. Within the manifold $J_{\mathrm{I}}=\sqrt{J_{\mathrm{H}}^{2}+D^{2}}$, the ground-state energy exhibits a rather striking nonanalytic behavior. Although this weak nonanalytic behavior cannot be seen from Fig. 2, it should manifest itself in higher derivatives of the ground-state energy.

\section{Conclusions}

In the present work, the ground-state properties of the spin-1/2 Heisenberg-Ising bond alternating chain with the Dzyaloshinskii-Moriya interaction have been investigated using a series of exact (rotation, Jordan-Wigner, Fourier, Bogolyubov) transformations. Exact results for the ground-state energy and elementary excitation spectrum have been examined in relation with a strength of the ratio between the Ising and Heisenberg interaction, as well as the Dzyaloshinskii-Moriya term. The most interesting finding to emerge from our study closely relates 
to a remarkable nonanalytic behavior of the ground-state energy, which is accompanied with the gapless excitation spectrum whenever the condition $J_{\mathrm{I}}=\sqrt{J_{\mathrm{H}}^{2}+D^{2}}$ is met.

\section{Acknowledgments}

J.S., L.G., and O.D. thank the Abdus Salam International Centre for Theoretical Physics (Trieste, Italy) for hospitality during the School and Workshop on Highly Frustrated Magnets and Strongly Correlated Systems: From Non-Perturbative Approaches to Experiments (2007), where the present study was launched. J.S. and L.G. acknowledge financial support provided under the grants VEGA 1/0128/08 and VEGA 1/0431/10.

\section{References}

[1] D.C. Mattis, The Many-Body Problem: An Encyclopedia of Exactly Solved Models in One Dimension, World Scientific, Singapore 1993.

[2] E. Lieb, T. Schultz, D. Mattis, Ann. Phys. 16, 407 (1961).

[3] H. Yao, J. Li, Ch.-D. Gong, Solid State Commun. 121, 687 (2002). 